\title{
CORRIGENDUM: Annulling a dangerous liaison: vaccination strategies against AIDS and tuberculosis
}

Stefan H E Kaufmann \& Andrew J McMichael

Nat. Med. 11 Suppl 4, S33-S44

The sentence "In South Africa, more than $10 \%$ of the 40 million inhabitants are infected with HIV and more than 5\% suffer from active tuberculosis" should read "more than $0.5 \%$ suffer from active tuberculosis."

In Table 2, line 3 should read "Clinical phase 1 trial in BCG-vaccinated and unvaccinated healthy uninfected volunteers completed".

\section{CORRIGENDUM: Interaction between leukemic-cell VLA-4 and stromal} fibronectin is a decisive factor for minimal residual disease of acute myelogenous leukemia

Takuya Matsunaga, Naofumi Takemoto, Tsutomu Sato, Rishu Takimoto, Ikuta Tanaka, Akihito Fujimi, Takehide Akiyama, Hiroyuki Kuroda, Yutaka Kawano, Masayoshi Kobune, Junji Kato, Yasuo Hirayama, Sumio Sakamaki, Kyuhei Kohda, Kensuke Miyake \& Yoshiro Niitsu Nat. Med.9, 1158-1165 (2003)

Two sentences in this article appeared incorrectly. On page 1164, in the eighth paragraph, the third sentence should read "To the U937-transplanted SCID mice, $1 \mathrm{mg}$ of either SG/17, YN907 or vehicle was injected intravenously, and $20 \mathrm{mg}$ of either AraC or normal saline was injected intraperitoneally on day 7 of transplantation." On page 1164, in the eighth paragraph, the fourth sentence should read “To NOD-SCID mice transplanted with patients' leukemic cells, 2 mg of either SG/17 or YN907 was injected intravenously, and $40 \mathrm{mg}$ of either AraC or normal saline was injected intraperioneally on days 3 and 4 of transplantation." 